\title{
On the possible association of SN 1998bw and GRB 980425
}

\author{
T.J. Galama ${ }^{1}$, P.M. Vreeswijk ${ }^{1}$, J. van Paradijs ${ }^{1,2}$, C. Kouveliotou ${ }^{3,4}$, T. Augusteijn ${ }^{5}$, F. Patat $^{5}$, J. Heise $^{6}$, \\ J. in 't Zand ${ }^{6}$, P.J. Groot ${ }^{1}$, R.A.M.J. Wijers ${ }^{7}$, E. Pian ${ }^{8}$, E. Palazzi ${ }^{8}$, F. Frontera ${ }^{8}$, and N. Masetti ${ }^{8}$ \\ 1 Astronomical Institute "Anton Pannekoek"/CHEAF, Kruislaan 403, 1098 SJ, Amsterdam, The Netherlands \\ 2 Physics Department, UAH, Huntsville, AL 35899, U.S.A. \\ 3 Universities Space Research Association, U.S.A. \\ 4 NASA Marshall Space Flight Center, ES-84, Huntsville, AL 35812, U.S.A. \\ 5 ESO, Casilla 19001, Santiago 19, Chile \\ 6 SRON Laboratory for Space Research, Sorbonnelaan 2, 3584 CA Utrecht, The Netherlands \\ 7 Dept. of Physics and Astronomy, SUNY, Stony Brook, NY 11794-3800, U.S.A. \\ 8 Istituto Tecnologie e Studio Radiazioni Extraterrestri, CNR, Bologna, Italy
}

Received December 29, 1998; accepted May 4, 1999

\begin{abstract}
We here discuss the possibility that the peculiar supernova SN 1998bw and the Gamma-Ray Burst GRB 980425 are associated. If the two are related, the energy radiated in $\gamma$ rays is at least four orders of magnitude less than in other GRBs, although its appearance is otherwise unremarkable: this suggests that very different mechanisms may give rise to GRBs.
\end{abstract}

Key words: gamma-rays: bursts - (stars:) supernovae: individual: SN 1998bw

\section{Introduction}

GRB 980425 was detected (Soffitta et al. 1998) on April 25.91 UT with one of the Wide Field Cameras (WFCs) and the Gamma Ray Burst Monitor (GRBM) on board BeppoSAX, and with the Burst and Transient Source Experiment (BATSE). With respect to its $\gamma$-ray properties, GRB 980425 was not a remarkable event (Galama et al. 1998).

SN 1998bw was discovered by inspection of New Technology Telescope (NTT) images and the Digitized Sky Survey (Galama et al. 1998). Its position at R.A. $=19^{\mathrm{h}} 35^{\mathrm{m}} 03.34^{\mathrm{s}} \pm 0.02^{\mathrm{s}}$, Decl. $=-52^{\circ} 50^{\prime} 44.8^{\prime \prime} \pm$ $0.2^{\prime \prime}(\mathrm{J} 2000.0), 1 ! 6$ away from the center of the $8^{\prime}$ radius WFC error box (Soffitta et al. 1998), is within the BATSE/Ulysses IPN annulus and coincides with that of the transient radio source in the WFC error box (Kulkarni et al. 1998) to within 0'.3 (see Fig. 1). It is located in an HII region in a spiral arm of the face-on

Send offprint requests to: T.J. Galama barred spiral galaxy ESO 184-G82 at a redshift of $2550 \mathrm{~km} \mathrm{~s}^{-1}$, in the DN 1931-529 group of galaxies (see Fig. 2).

The GRB and the supernova are spatially and temporally coincident; the time of occurrence of the core collapse and the GRB coincide to within $(+0.7,-2.0)$ days (Iwamoto et al. 1998). Any estimate of the probability that the supernova and the GRB coincided by chance (with respect to both time and direction) suffers from the problem of a posteriori statistics, i.e., that the parameters of the problem tend to be set by the observed phenomenon itself. In this case the parameters are the size of the error box, the peak magnitude of the supernova, and the time window within which the events can be considered as possibly related. Galama et al. (1998) made generous estimates of these parameters and find the probability of catching a $\mathrm{SN}$ in any of the $13 \mathrm{WFC}$ GRB error boxes to be $910^{-5}$. This estimate includes all supernovae with peak magnitudes two magnitudes below that of SN 1998bw, and ignores the fact that SN 1998bw is of a rare type. As a result, the notion that GRB 980425 and SN 1998bw are physically related becomes difficult to reject purely on the basis of the fact that afterglows observed so far from GRBs are very different from supernovae.

\section{X-ray sources}

The WFC error box contains two X-ray sources (Pian et al. 1998a,b), one of which coincides with SN 1998bw (Piro et al. 1998). The other one, 1SAX J1935.3 - 5252,

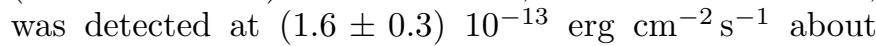
1 day after the burst, and was marginally detected 6 days

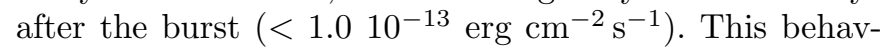
ior is inconsistent with that of previously observed X-ray 

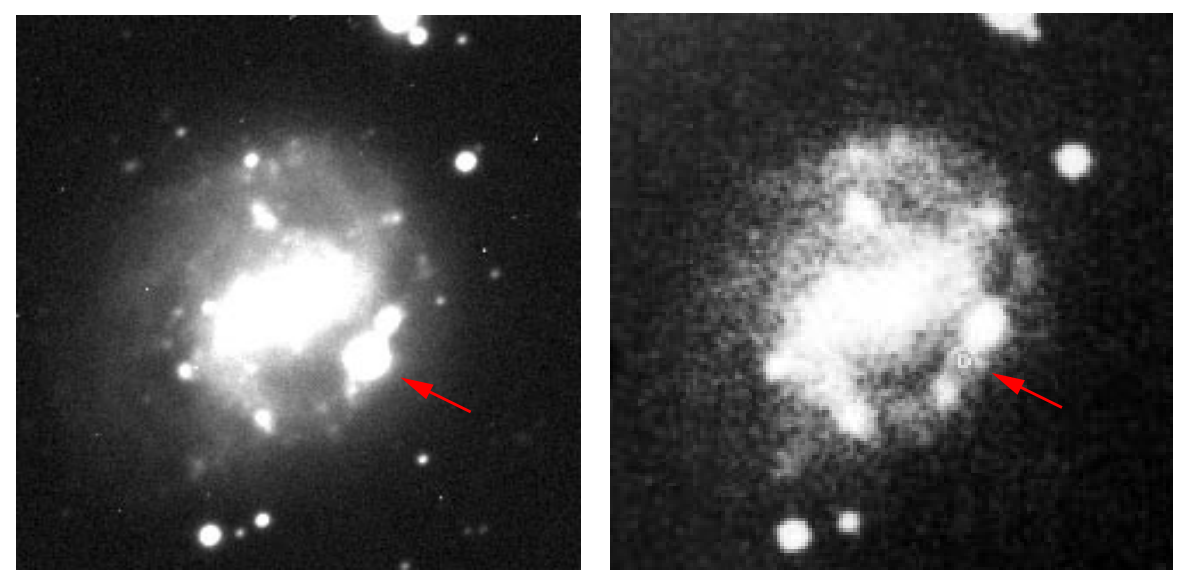

Fig. 2. Image on the left: NTT image (May 1 1998) of SN 1998bw in the barred spiral galaxy ESO 184-G82. Image on the right: Pre-discovery image from 1976 (UK Schmidt Telescope, Australia)

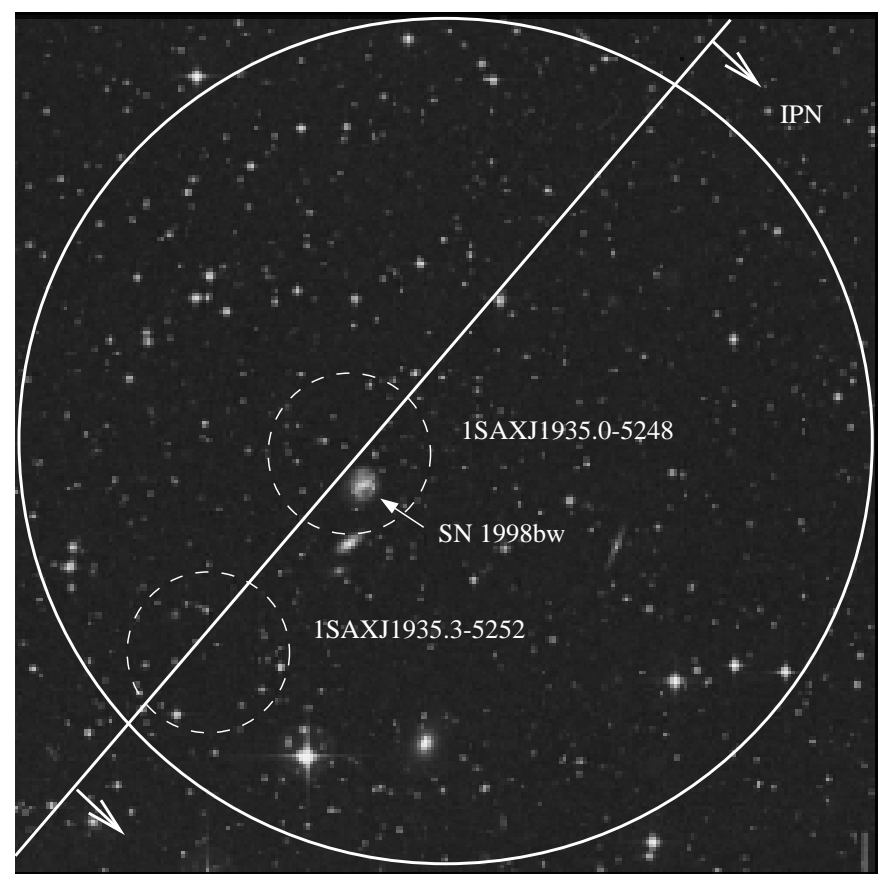

Fig. 1. Digitized Sky Survey image $\left(16^{\prime} \times 16^{\prime}\right)$ of the WFC error box of GRB 980425 (large circle of radius $8^{\prime}$ ). The left boundary of the IPN annulus is indicated as well as the error boxes of the two NFI X-ray sources (dashed circles of radius $90^{\prime \prime}$ ) and the position of SN 1998bw. The two X-ray sources as well as the position of SN 1998bw are consistent with the WFC and IPN locations

afterglows of GRBs, although it could be reconciled with it under some assumption (see the contribution to these proceedings by Pian et al.).

The $2-10 \mathrm{keV}$ detection limit $(3 \sigma)$ for the GRB 980425 Narrow-Field Instrument observations was $1.210^{-13} \mathrm{erg} \mathrm{s}^{-1} \mathrm{~cm}^{-2}$. Using the ASCA $2-10 \mathrm{keV}$ source count distributions (Cagnoni et al. 1998) one expects to find an average of $0.6 \mathrm{X}$-ray sources above this limit in the WFC error box; the probability of finding two or more sources there by chance coincidence is $12 \%$. The case for a relation between this X-ray source and GRB 980425 must therefore be considered tentative at best, in particular since among weak ROSAT sources variability is not rare.

\section{Discussion}

The consequence of an association is that the $\gamma$-ray peak luminosity of GRB 980425 is $L_{\gamma}=(5.5 \pm 0.7) 10^{46} \mathrm{erg}$ $\mathrm{s}^{-1}(24-1820 \mathrm{keV})$ and its total $\gamma$-ray energy budget $(8.1 \pm 1.0) 10^{47} \mathrm{erg}$ (assuming isotropic emission). These values are much smaller than those of "normal" GRBs which have peak luminosities of up to $10^{52} \mathrm{erg} \mathrm{s}^{-1}$ and total energies up to several times $10^{53} \mathrm{erg}$. It is then likely that very different mechanisms can produce GRBs which cannot be distinguished on the basis of their $\gamma$-ray properties, and that models explaining GRB 980425/SN 1998bw are unlikely to apply to "normal" GRBs and vice versa. However, searches for correlations between GRBs and supernovae do not find evidence for such a relation but allow that a fraction of Ib/Ic-type supernovae produce a GRB (Kippen et al. 1998; Graziani et al. 1999).

\section{References}

Cagnoni I., Della Ceca R., Maccacaro T., 1998, AJ 493, 54 Galama T.J., et al., 1998, Nat 395, 670

Graziani C., Lamb D.Q., Marion G.H., 1999, ApJ (submitted) Iwamoto K., et al., 1998, Nat 395, 672

Kippen R.M., et al., 1998, ApJL 506, L27

Kulkarni S.R., et al., 1998, Nat 395, 663

Pian E., et al., 1998a, GCN Circ. 61

Pian E., et al., 1998b, GCN Circ. 69

Piro L., et al., 1998, GCN Circ. 155

Soffitta P., et al., 1998, IAU Circ., 6884 